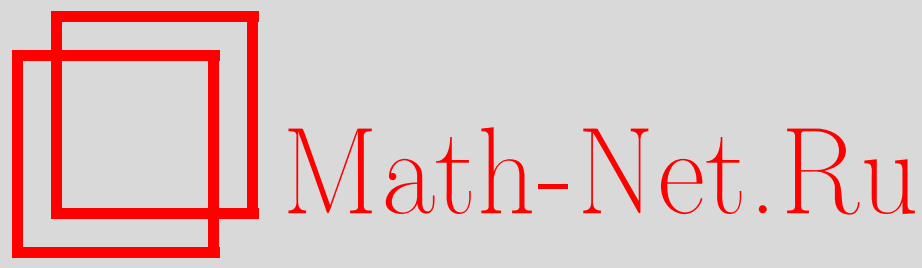

Дж. Сколаричи, Замечания об альтернативных эрмитовых структурах для составных квантовых систем, $Т M \Phi$, 2009, том 160, номер 1, 211-219

DOI: https://doi.org/10.4213/tmf6392

Использование Общероссийского математического портала Math-Net.Ru подразумевает, что вы прочитали и согласны с пользовательским соглашением http://www.mathnet.ru/rus/agreement

Параметры загрузки:

IP: 54.209 .52 .79

26 апреля 2023 г., 10:56:00

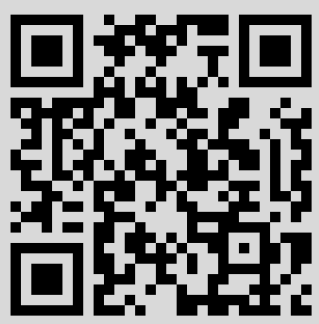




\title{
ФИЗИКА
}

Том 160, № 1

июль, 2009

\section{ЗАМЕЧАНИЯ ОБ АЛЬТЕРНАТИВНЫХ ЭРМИТОВЫХ СТРУКТУРАХ ДЛЯ СОСТАВНЫХ КВАНТОВЫХ СИСТЕМ}

\begin{abstract}
Приводятся некоторые замечания, касающиеся квантовых систем, которые состоят из двух частей и обладают альтернативными эрмитовыми структурами на бесконечномерных комплексных гильбертовых пространствах.
\end{abstract}

Ключевые слова: биунитарные преобразования, квантовые бигамильтоновы системы, альтернативные эрмитовы структуры, запутанность.

\section{1. ВВЕДЕНИЕ}

Хорошо известно, что значительное число нелинейных эволюционных уравнений являются интегрируемыми системами [1]. Почти во всех случаях интегрируемые системы допускают альтернативное гамильтоново описание и называются бигамильтоновыми системами [2]. В целом ряде работ [3]-[16] было показано, что аналог альтернативного гамильтонова описания можно реализовать в рамках квантовой теории. В этом контексте интересно проанализировать, насколько подобное альтернативное квантовое описание окажется применимым при рассмотрении составных систем и взаимодействий между ними [10]. В настоящей работе представлены некоторые замечания, касающиеся этого анализа. В частности, показано, что составные квантовые системы, наделенные альтернативными эрмитовыми структурами на одном и том же бесконечномерном комплексном векторном пространстве, допускают подходящее описание в терминах составляющих систем тогда и только тогда, когда положительный оператор, который связывает альтернативное скалярное произведение с опорным, можно записать как тензорное произведение положительных операторов в составляющих пространствах. Более того, показано, что энтропия фон Неймана, связанная со смешанными состояниями оператора плотности, в сильной степени зависит от альтернативных эрмитовых структур.

${ }^{*}$ Dipartimento di Fisica dell'Università del Salento; INFN, Sezione di Lecce, Lecce, Italy. E-mail: scolarici@le.infn.it 
Работа построена следующим образом. В разделе 2, после краткого описания биунитарной группы на бесконечномерном комплексном гильбертовом пространстве, показано, что альтернативные эрмитовы структуры отвечают разным средним биэрмитовых наблюдаемых; в частности, показано, что энтропия фон Неймана сильно зависит от альтернативного скалярного произведения. В разделе 3 установлено необходимое и достаточное условие для того, чтобы составные квантовые системы, наделенные альтернативными эрмитовыми структурами, можно было рассматривать в терминах составляющих систем на бесконечномерном гильбертовом пространстве (см. предложения 2 и 3). Более того, доказано необходимое условие того, что альтернативная эрмитова структура, связанная с составляющими системами, находится в общем положении по отношению к опорной (см. предложение 1). В разделе 4 рассматривается простой пример, в разделе 5 приведены заключительные замечания.

\section{2. БИУНИТАРНЫЕ ОПИСАНИЯ НА ГИЛЬБЕРТОВОМ ПРОСТРАНСТВЕ}

Геометрический подход к квантовой механике основан на овеществлениях $\mathcal{H}^{\mathbb{R}}$ комплексного гильбертова пространства $\mathcal{H}$. В квантовой механике комплексная структура входит непосредственно в уравнение Шредингера, поэтому естественно рассматривать вещественные гильбертовы пространства $\mathcal{H}^{\mathbb{R}}$, возникающие при одном и том же овеществлении пространства $\mathcal{H}$. В настоящей работе мы будем рассматривать две эрмитовы структуры $h_{1}=g_{1}+i \omega_{1}$ и $h_{2}=g_{2}+i \omega_{2}$ на пространстве $\mathcal{H}$, происходящие, разумеется, из двух допустимых троек $\left(g_{1}, J_{1}, \omega_{1}\right)$ и $\left(g_{2}, J_{2}, \omega_{2}\right)$ на $\mathcal{H}^{\mathbb{R}}[10]$, причем мы предполагаем, что комплексные структуры совпадают, $J_{1}=J_{2}=J$.

В случае, когда $J_{1}=J_{2}$, теорема Ритца обеспечивает выполнение следующего соотношения для $h_{1}$ и $h_{2}$ :

$$
h_{2}(x, y)=h_{1}(F x, y) \quad \forall x, y \in \mathcal{H},
$$

где $F$ - ограниченный положительный самосопряженный оператор по отношению к обеим эрмитовым структурам, причем он содержит как вещественную, так и мнимую части $h_{1}$ и $h_{2}$ :

$$
F=\left(g_{1}+i \omega_{1}\right)^{-1} \circ\left(g_{2}+i \omega_{2}\right) .
$$

Любой биунитарный оператор $U$ должен коммутировать с $F$, поэтому группа биунитарных операторов принадлежит коммутанту $F^{\prime}$ оператора $F$ :

$$
U^{\dagger} F U=F, \quad[F, U]=0 .
$$

Более того, $h_{1}$ и $h_{2}$ определяют два представления $U\left(\mathcal{H}, h_{a}\right), a=1,2$, унитарной группы $U(\mathcal{H})$ на том же гильбертовом пространстве $\mathcal{H}$ (здесь $U\left(\mathcal{H}, h_{a}\right)$ обозначает группу преобразований, унитарных относительно $\left.h_{a}\right)$. Тогда группа биунитарных преобразований является подгруппой как группы $U\left(\mathcal{H}, h_{1}\right)$, так и группы $U\left(\mathcal{H}, h_{2}\right)$, принадлежащей коммутанту $F^{\prime}$ оператора $F$ :

$$
\{U\}=U\left(\mathcal{H}, h_{1}\right) \cap U\left(\mathcal{H}, h_{2}\right) .
$$


Обозначим через $H\left(\mathcal{H}, h_{a}\right), a=1,2$, множества эрмитовых операторов относительно $h_{a}$. Множество биэрмитовых операторов $H$, т.е. эрмитовых операторов относительно $h_{1}$ и $h_{2}$, является подмножеством как в $H\left(\mathcal{H}, h_{1}\right)$, так и в $H\left(\mathcal{H}, h_{2}\right)$ :

$$
\{H\}=H\left(\mathcal{H}, h_{1}\right) \cap H\left(\mathcal{H}, h_{2}\right) .
$$

Опишем явно биунитарную группу в бесконечномерном случае [16]. Связывающий оператор $F$ может иметь в своем спектре как точечную, так и непрерывную части. Что касается точечной части, то биунитарная группа есть $U\left(n_{1}\right) \times U\left(n_{2}\right) \times \cdots$ $\cdots \times U\left(n_{k}\right) \times \cdots$, где вырождения $n_{k}$ собственных значений оператора $F$ могут быть также равными бесконечности. В отношении непрерывной части напомним [16], что (слабозамкнутое коммутативное) кольцо $R(F)$, порожденное оператором $F$, индуцирует разложение гильбертова пространства $\mathcal{H}$ в прямой интеграл

$$
\mathcal{H}=\bigoplus_{k} \int_{\Delta_{k}} \mathcal{H}_{\lambda} d \sigma(\lambda),
$$

где полный спектр $\Delta$ оператора $F$ представляет собой объединение счетного числа измеримых множеств $\Delta_{k}$ таких, что при $\lambda \in \Delta_{k}$ пространства $\mathcal{H}_{\lambda}$ имеют одну и ту же размерность $n_{k}$ (возможно, бесконечную), а мера $\sigma(\lambda)$ получается обычным путем при использовании спектрального семейства $\left\{P_{F}(\lambda)\right\}$ оператора $F$ и циклических векторов [17].

Элементы $U$ действующей на $\mathcal{H}$ биунитарной группы имеют вид [16]

$$
U=\bigoplus_{k} \int_{\Delta_{k}} U_{n_{k}}(\lambda) d \sigma(\lambda),
$$

где $U_{n_{k}}(\lambda)$ - элемент унитарной группы $U\left(n_{k}\right)$ при каждом $\lambda \in \Delta_{k}$. Более того, поскольку $F$ действует как оператор умножения на каждой компоненте пространства $\mathcal{H}_{\lambda}$, выражения для $h_{1}$ и $h_{2}$ на $\mathcal{H}$ имеют вид

$$
\begin{aligned}
& h_{1}(x, y)=\sum_{k} \int_{\Delta_{k}}\left\langle x_{\lambda} \mid y_{\lambda}\right\rangle d \sigma(\lambda), \\
& h_{2}(x, y)=\sum_{k} \int_{\Delta_{k}} \lambda\left\langle x_{\lambda} \mid y_{\lambda}\right\rangle d \sigma(\lambda),
\end{aligned}
$$

где $\left\langle x_{\lambda} \mid y_{\lambda}\right\rangle$ - скалярное произведение на $\mathcal{H}_{\lambda}$.

Две эрмитовы структуры находятся в общем положении, если бикоммутант $F^{\prime \prime}$ оператора $F$ совпадает с его коммутантом $F^{\prime}[10]$ или, что эквивалентно, если оператор $F$ является циклическим на $\mathcal{H}[16]$. В этом случае биунитарная группа сводится к умножению на фазовый множитель $e^{i \vartheta(\lambda)}$ на одномерном пространстве $\mathcal{H}_{\lambda}$ для почти каждого $\lambda$ :

$$
U=\int_{\Delta} e^{i \vartheta(\lambda)} d \sigma(\lambda)
$$

Поэтому в более актуальном случае общего положения, который соответствует полной интегрируемости квантовой системы, группа биунитарных преобразований параметризуется $\sigma$-измеримыми вещественными функциями $\vartheta$ на $\Delta$, а следовательно, 
можно записать

$$
U_{\vartheta}=e^{i \vartheta(F)} .
$$

В этом случае биэрмитов гамильтониан имеет вид

$$
H_{\vartheta}=\vartheta(F)
$$

и однопараметрическую биунитарную группу эволюции можно записать в обычном виде:

$$
U(t)=e^{-i H_{\vartheta} t}
$$

Используя альтернативное скалярное произведение (9), среднее значение биэрмитова оператора $H$ в (нормированном) состоянии можно записать как

$$
\begin{aligned}
\frac{h_{2}(x, H x)}{h_{2}(x, x)} & =\frac{1}{h_{2}(x, x)} \sum_{k} \int_{\Delta_{k}} \lambda\left\langle x_{\lambda}|H| x_{\lambda}\right\rangle d \sigma(\lambda)=\frac{1}{h_{2}(x, x)} \sum_{k} \int_{\Delta_{k}}\left\langle F x_{\lambda}|H| x_{\lambda}\right\rangle d \sigma(\lambda)= \\
& =\frac{1}{h_{2}(x, x)} \sum_{k} \int_{\Delta_{k}}\left|x_{\lambda}\right\rangle\left\langle F x_{\lambda}\right| H d \sigma(\lambda)=\operatorname{Tr}\left(\frac{|x\rangle\langle F x|}{\langle F x \mid x\rangle} H\right)=\operatorname{Tr}\left(\rho_{F} H\right), \quad(13)
\end{aligned}
$$

где $\operatorname{Tr}$ обозначает операцию взятия следа, а

$$
\rho_{F}=\frac{|x\rangle\langle F x|}{\langle F x \mid x\rangle}
$$

представляет собой оператор плотности ранга 1 в новом описании. Взяв выпуклую комбинацию проекторов ранга 1, мы можем построить всю совокупность состояний, связанных с оператором плотности, которую мы также обозначим как $\rho_{F}$. В конечномерном случае геометрия таких состояний очевидна, если рассматривается стандартная эрмитова структура [18]-[20].

Легко проверить, что динамика оператора $\rho_{F}$ определяется на инфинитезимальном уровне уравнением Лиувилля-фон Неймана

$$
\frac{d}{d t} \rho_{F}(t)=-i\left[H_{\vartheta}, \rho_{F}\right]
$$

Ясно, что изменение скалярного произведения соответствует различным средним значениям оператора $H$ в одном и том же состоянии. На самом деле $\rho_{F}$ и $\rho$ представляют одно и то же (смешанное) состояние в двух разных описаниях, но, вообе говоря, они имеют различные спектры, так что

$$
\langle H\rangle_{2}=\operatorname{Tr}\left(\rho_{F} H\right) \neq \operatorname{Tr}(\rho H)=\langle H\rangle_{1} .
$$

В частности, обозначая через $S$ энтропию фон Неймана, связанную с оператором плотности, мы немедленно получаем

$$
S\left(\rho_{F}\right)=-\operatorname{Tr}\left(\rho_{F} \ln \rho_{F}\right) \neq-\operatorname{Tr}(\rho \ln \rho)=S(\rho),
$$

а потому энтропия фон Неймана зависит от альтернативного скалярного произведения, которое мы можем использовать. Тогда можно заключить, что энтропия и среднее значение биэрмитовых наблюдаемых сильно зависят от альтернативного описания, рассматриваемого на $\mathcal{H}$, и поэтому альтернативные описания могут представлять различные физические теории, если только мы не обнаружим различные способы отождествления математических сущностей с физическими величинами. 


\section{3. АЛЬТЕРНАТИВНЫЕ ОПИСАНИЯ СОСТАВНЫХ КВАНТОВЫХ СИСТЕМ}

Перейдем к составным системам. Предположим, что два гильбертовых пространства $\mathcal{H}_{1}$ и $\mathcal{H}_{2}$ (размерности которых могут равняться бесконечности) наделены альтернативными эрмитовыми структурами, соответственно задаваемыми с помощью $h_{F_{1}}(\cdot, \cdot)=h\left(\cdot, F_{1} \cdot\right)$ и $h_{F_{2}}(\cdot, \cdot)=h\left(\cdot, F_{2} \cdot\right)$, где $h$ обозначает опорное скалярное произведение в $\mathcal{H}_{1}$ и $\mathcal{H}_{2}$.

Отображение тензорного произведения

$$
\otimes: \mathcal{H}_{1} \times \mathcal{H}_{2} \rightarrow \mathcal{H}=\mathcal{H}_{1} \otimes \mathcal{H}_{2}
$$

переводит произведение лучей в луч, так что оно индуцирует каноническое вложение на уровне комплексных проективных пространств [19], [20]

$$
\begin{gathered}
\text { Seg: } \mathcal{P H}_{1} \times \mathcal{P} \mathcal{H}_{2} \rightarrow \mathcal{P H}=\mathcal{P}\left(\mathcal{H}_{1} \otimes \mathcal{H}_{2}\right), \\
\left(\frac{\left|x_{1}\right\rangle\left\langle F_{1} x_{1}\right|}{\left\langle F_{1} x_{1} \mid x_{1}\right\rangle}, \frac{\left|x_{2}\right\rangle\left\langle F_{2} x_{2}\right|}{\left\langle F_{2} x_{2} \mid x_{2}\right\rangle}\right) \mapsto \frac{\left|x_{1} \otimes x_{2}\right\rangle\left\langle F_{1} x_{1} \otimes F_{2} x_{2}\right|}{\left\langle F_{1} x_{1} \otimes F_{2} x_{2} \mid x_{1} \otimes x_{2}\right\rangle} .
\end{gathered}
$$

Это вложение связано с тензорным произведением основных представлений унитарных групп $U\left(\mathcal{H}_{1}, h_{F_{1}}\right)$ и $U\left(\mathcal{H}_{2}, h_{F_{2}}\right)$, т.е. с представлением прямого произведения групп на $\mathcal{H}=\mathcal{H}_{1} \otimes \mathcal{H}_{2}$,

$$
\begin{aligned}
U\left(\mathcal{H}_{1}, h_{F_{1}}\right) \times U\left(\mathcal{H}_{2}, h_{F_{2}}\right) & \ni\left(U_{1}, U_{2}\right) \mapsto U_{1} \otimes U_{2} \in U\left(\mathcal{H}, h_{F_{1} \otimes F_{2}}\right), \\
\left(U_{1}, U_{2}\right)\left(x_{1} \otimes x_{2}\right) & =U_{1}\left(x_{1}\right) \otimes U_{2}\left(x_{2}\right) .
\end{aligned}
$$

Группа $U_{1} \otimes U_{2}$ является унитарной, поскольку эрмитово произведение $h_{F_{1} \otimes F_{2}}$ в $\mathcal{H}$ связано с эрмитовыми произведениями $h_{F_{1}}$ и $h_{F_{2}}$ в $\mathcal{H}_{1}$ и $\mathcal{H}_{2}$ следующим образом:

$$
h_{F_{1} \otimes F_{2}}\left(x_{1} \otimes x_{2}, y_{1} \otimes y_{2}\right)=h_{F_{1}}\left(x_{1}, y_{1}\right) h_{F_{2}}\left(x_{2}, y_{2}\right) \text {. }
$$

Приведенное выше групповое вложение порождает соответствующее вложение их групповых алгебр, что мы обозначим как

$$
\begin{aligned}
H\left(\mathcal{H}_{1}, h_{F_{1}}\right) \times H\left(\mathcal{H}_{2}, h_{F_{2}}\right) & \rightarrow H\left(\mathcal{H}, h_{F_{1} \otimes F_{2}}\right), \\
\left(H_{1}, H_{2}\right) & \mapsto H_{1} \otimes H_{2} .
\end{aligned}
$$

Следующее предложение дает необходимое условие того, чтобы альтернативная эрмитова структура, связанная с составной системой, находилась в общем относительном положении по отношению к опорной.

ПредЛОЖениЕ 1. Альтернативная структура $h_{F_{1} \otimes F_{2}}$ находится в общем положении на $\mathcal{H}$, только если $h_{F_{1}}$ и $h_{F_{2}}$ находятся в общем относительном положении на $\mathcal{H}_{1}$ и $\mathcal{H}_{2}$ соответственно.

ДоКАЗАТЕЛЬСТво. Действуя от противного, предположим, что бикоммутант $F_{1}^{\prime \prime}$ оператора $F_{1}$ (или бикоммутант $F_{2}^{\prime \prime}$ оператора $F_{2}$ ) отличается от его коммутанта $F_{1}^{\prime}\left(F_{2}^{\prime}\right)$. Тогда имеется некоторый элемент из множества $\left\{f_{1}^{\prime \prime} \otimes f_{2}^{\prime \prime}\right\}$, где $f_{1}^{\prime \prime} \in F_{1}^{\prime \prime}$ 
и $f_{2}^{\prime \prime} \in F_{2}^{\prime \prime}$, который не может принадлежать множеству $\left\{f_{1}^{\prime} \otimes f_{2}^{\prime}\right\}$, где $f_{1}^{\prime} \in F_{1}^{\prime}$ и $f_{2}^{\prime} \in F_{2}^{\prime}$, но $\left\{f_{1}^{\prime \prime} \otimes f_{2}^{\prime \prime}\right\} \subset\left(F_{1} \otimes F_{2}\right)^{\prime \prime}$ и $\left\{f_{1}^{\prime} \otimes f_{2}^{\prime}\right\} \subset\left(F_{1} \otimes F_{2}\right)^{\prime}$. Тогда немедленно получаем $\left(F_{1} \otimes F_{2}\right)^{\prime \prime} \neq\left(F_{1} \otimes F_{2}\right)^{\prime}$.

Если эрмитовы структуры $h_{F_{1}}$ и $h_{F_{2}}$ находятся в общих относительных положениях (по отношению к опорным) на $\mathcal{H}_{1}$ и $\mathcal{H}_{2}$ соответственно, а $F_{1}$ и $F_{2}$ допускают дискретные спектры,

$$
\begin{array}{ll}
F_{1}=\sum_{i} r_{i}\left|r_{i}\right\rangle\left\langle r_{i}\right|, \quad & r_{i}>0, \\
F_{2}=\sum_{j} s_{j}\left|s_{j}\right\rangle\left\langle s_{j}\right|, & s_{j}>0,
\end{array}
$$

то редуцированные операторы плотности, связанные с оператором

$$
\rho_{F_{1} \otimes F_{2}}=\frac{|x\rangle\left\langle\left(F_{1} \otimes F_{2}\right) x\right|}{\left\langle\left(F_{1} \otimes F_{2}\right) x \mid x\right\rangle} \in \mathcal{P} \mathcal{H}=\mathcal{P}\left(\mathcal{H}_{1} \otimes \mathcal{H}_{2}\right),
$$

можно получить обычным способом, вычисляя частичные следы:

$$
\begin{aligned}
& \rho_{F_{1}}=\operatorname{Tr}_{2}\left(\rho_{F_{1} \otimes F_{2}}\right)=\sum_{j}\left\langle s_{j}\left|\rho_{F_{1} \otimes F_{2}}\right| s_{j}\right\rangle, \\
& \rho_{F_{2}}=\operatorname{Tr}_{1}\left(\rho_{F_{1} \otimes F_{2}}\right)=\sum_{i}\left\langle r_{i}\left|\rho_{F_{1} \otimes F_{2}}\right| r_{i}\right\rangle .
\end{aligned}
$$

ЗАмечАниЕ. Очевидно, что если $F=F_{1} \otimes F_{2}$, то свойство состояния, связанного с составными системами, быть или не быть запутанным не зависит от альтернативных структур $h_{F_{1}}$ и $h_{F_{2}}$ на составляющих пространствах. Действительно, рассмотрим состояние $|z\rangle=|x\rangle \otimes|y\rangle$. Состояние $\left|z^{\prime}\right\rangle=S|z\rangle$ становится запутанным тогда и только тогда, когда $S \in G L(\mathcal{H})$ и $S \neq S_{1} \otimes S_{2}$ для любого $S_{1} \in G L\left(\mathcal{H}_{1}\right)$ и $S_{2} \in G L\left(\mathcal{H}_{2}\right)$ [19], [20]. Поэтому (альтернативная) эрмитова структура не играет никакой роли.

Рассмотрим обратную задачу. Предположим, что на бесконечномерном гильбертовом пространстве $\mathcal{H}$ задана однопараметрическая биунитарная групповая эволюция (12), связанная с составными квантовыми системами и оставляющая инвариантной альтернативную эрмитову структуру $h_{F}(\cdot, \cdot)=h(\cdot, F \cdot)$. Тогда возникает вопрос: возможно ли чисто квантово-механическое описание таких составных квантовых систем в терминах составляющих их систем, если рассматривается альтернативная эрмитова структура?

Следующее предложение дает необходимое и достаточное условие того, чтобы гильбертовы пространства $\mathcal{H}_{1}$ и $\mathcal{H}_{2}$, связанные с составляющими системами, были наделены подходящими альтернативными эрмитовыми структурами $h_{F_{1}}$ и $h_{F_{2}}$ такими, что $\forall\left(U_{1}, U_{2}\right) \in U\left(\mathcal{H}_{1}, h_{F_{1}}\right) \times U\left(\mathcal{H}_{2}, h_{F_{2}}\right)$

$$
U_{1} \otimes U_{2} \in U\left(\mathcal{H}, h_{F}\right) .
$$

ПреДЛОЖЕНИЕ 2. Группа $U\left(\mathcal{H}, h_{F}\right)$ содержит преобразования $U_{1} \otimes U_{2}$ для любъх $U_{1} \in U\left(\mathcal{H}_{1}, h_{F_{1}}\right)$ и $U_{2} \in U\left(\mathcal{H}_{2}, h_{F_{2}}\right)$ тогда и только тогда, когда $F=F_{1} \otimes F_{2}$. 
Доказательство предложения 2 легко получить, если переписать это утверждение в терминах соответствующей групповой алгебры.

ПреДЛОЖЕНИЕ 3. Множество $H\left(\mathcal{H}, h_{F}\right)$ содержит операторъ $H_{1} \otimes H_{2}$ для любъих $H_{1} \in H\left(\mathcal{H}_{1}, h_{F_{1}}\right)$ и $H_{2} \in H\left(\mathcal{H}_{2}, h_{F_{2}}\right)$ тогда и только тогда, когда $F=F_{1} \otimes F_{2}$.

ДокАЗАТЕЛЬСтво. Предположим, что $F=F_{1} \otimes F_{2}$. Тогда тривиальным образом имеем $H_{1} \otimes H_{2} \in H\left(\mathcal{H}, h_{F}\right)$ для любых $H_{1} \in H\left(\mathcal{H}_{1}, h_{F_{1}}\right)$ и $H_{2} \in H\left(\mathcal{H}_{2}, h_{F_{2}}\right)$. Обратно, предположим, что $F \neq F_{1} \otimes F_{2}$ для любых положительных операторов $F_{1}$ и $F_{2}$. Тогда множество $\left\{H_{1} \otimes H_{2}\right\}$ при всех $H_{1} \in H\left(\mathcal{H}_{1}, h_{F_{1}}\right)$ и $H_{2} \in H\left(\mathcal{H}_{2}, h_{F_{2}}\right)$, очевидно, является неприводимым множеством эрмитовых операторов в пространстве $\mathcal{H}$, наделенном эрмитовой структурой $h_{F_{1} \otimes F_{2}}$, поэтому оператор $F_{1} \otimes F_{2}$ является единственным (с точностью до нормировочного множителя) [21]. Тогда множество $\left\{H_{1} \otimes H_{2}\right\}$ содержит некоторый оператор, который не может быть эрмитовым по отношению к альтернативной эрмитовой структуре $h_{F}$.

Прямое следствие предложения 3 заключается в том, что тензорное произведение двух наблюдаемых в $H\left(\mathcal{H}_{1}, h_{F_{1}}\right)$ и $H\left(\mathcal{H}_{2}, h_{F_{2}}\right)$, несомненно, является наблюдаемой в $H\left(\mathcal{H}, h_{F}\right)$ тогда и только тогда, когда $F=F_{1} \otimes F_{2}$. Тогда можно заключить, что любое альтернативное описание квантовых систем, состоящих из двух частей, допускает чисто квантово-механическую интерпретацию в терминах составляющих их систем тогда и только тогда, когда $F=F_{1} \otimes F_{2}$.

Более того, если задан некоторый оператор $F$, допускающий разложение $F=$ $F_{1} \otimes F_{2}$, то такое разложение не является единственным. Действительно, можно, например, изменить операторы $F_{1}$ и $F_{2}$, умножив их на (положительные) множители $r$ и $1 / r$ соответственно.

\section{4. ПРОСТОЙ ПРИМЕР}

Теперь, ввиду актуальности двухкубитовых квантовых вентилей в квантовой теории информации и в области квантовых компьютеров, рассмотрим альтернативные описания для порождения оптимальной запутанности, недавно введенной в литературе [22]. Рассматриваемая система состоит из двух кубитов, поэтому $\mathcal{H} \equiv \mathbb{C}^{4}$. Оператор эволюции всей системы имеет вид

$$
U(t)=\left(\begin{array}{cccc}
e^{-i t} & 0 & 0 & 0 \\
0 & e^{i t} & 0 & 0 \\
0 & 0 & e^{i t} & 0 \\
0 & 0 & 0 & e^{-i t}
\end{array}\right)
$$

Пусть начальное состояние есть

$$
|x\rangle=\frac{1}{\sqrt{2}}\left(\begin{array}{l}
1 \\
1
\end{array}\right) \otimes \frac{1}{\sqrt{2}}\left(\begin{array}{l}
1 \\
i
\end{array}\right) .
$$


Рассмотрим в гильбертовом пространстве, связанном с составной системой, альтернативное скалярное произведение, связанное с опорным посредством (положительного) оператора,

$$
F=F_{1} \otimes F_{2}=\left(\begin{array}{cc}
r_{1} & 0 \\
0 & r_{2}
\end{array}\right) \otimes\left(\begin{array}{cc}
s_{1} & 0 \\
0 & s_{2}
\end{array}\right) .
$$

Заметим, что если $r_{1} \neq r_{2}, s_{1} \neq s_{2}$, то альтернативную эрмитову структуру $h_{F}$ на $\mathcal{H}$ можно выбрать в общем относительном положении в соответствии с предложением 1.

Исходный оператор плотности по отношению к $h_{F}$ имеет вид

$$
\rho_{F}(0)=\frac{|x\rangle\langle F x|}{\operatorname{Tr}|x\rangle\langle F x|},
$$

в момент времени $t$ получаем

$$
\rho_{F}(t)=U(t) \rho_{F}(0) U(t)^{\dagger}
$$

и из соотношений (19) можно вычислить редуцированные операторы плотности:

$$
\begin{aligned}
& \rho_{F_{1}}(t)=\operatorname{Tr}_{2}\left(\rho_{F}(t)\right)=\frac{1}{r_{1}+r_{2}}\left(\begin{array}{cc}
r_{1} & r_{2} \cos (2 t) \\
r_{1} \cos (2 t) & r_{2}
\end{array}\right), \\
& \rho_{F_{2}}(t)=\operatorname{Tr}_{1}\left(\rho_{F}(t)\right)=\frac{1}{s_{1}+s_{2}}\left(\begin{array}{cc}
s_{1} & -i s_{2} \cos (2 t) \\
i s_{1} \cos (2 t) & s_{2}
\end{array}\right) .
\end{aligned}
$$

Собственные значения оператора $\rho_{F_{1}}(t)$ имеют вид

$$
r_{ \pm}=\frac{1}{2}\left(1 \pm \frac{\sqrt{r_{1}^{2}+r_{2}^{2}+2 r_{1} r_{2} \cos (4 t)}}{r_{1}+r_{2}}\right),
$$

отсюда соответствующая энтропия фон Неймана имеет вид

$$
S\left(\rho_{F_{1}}(t)\right)=-\operatorname{Tr}\left(\rho_{F_{1}}(t) \ln \rho_{F_{1}}(t)\right)=-r_{+} \ln r_{+}-r_{-} \ln r_{-},
$$

откуда немедленно получаем, что энтропия зависит от альтернативного скалярного произведения.

Тогда мера запутывания сильно зависит от альтернативной эрмитовой структуры. Однако $S\left(\rho_{F_{1}}(t)\right)=0$, если степень чистоты обеих редуцированных матриц плотности

$$
\begin{aligned}
& P_{\rho_{F_{1}}(t)}=\operatorname{Tr}\left(\rho_{F_{1}}(t)^{2}\right)=\frac{1}{2}\left(1+\frac{r_{1}^{2}+r_{2}^{2}+2 r_{1} r_{2} \cos (4 t)}{\left(r_{1}+r_{2}\right)^{2}}\right), \\
& P_{\rho_{F_{2}}(t)}=\operatorname{Tr}\left(\rho_{F_{2}}(t)^{2}\right)=\frac{1}{2}\left(1+\frac{s_{1}^{2}+s_{2}^{2}+2 s_{1} s_{2} \cos (4 t)}{\left(s_{1}+s_{2}\right)^{2}}\right)
\end{aligned}
$$

становится равной единице, что имеет место, если $t=k \pi / 2, k \in \mathbb{N}$, независимо от матричных элементов $r_{1}, r_{2}, s_{1}$ и $s_{2}$.

Из равенств (26) заключаем, что, тогда как энтропия зависит от альтернативного скалярного произведения, свойство состояния быть или не быть запутанным не зависит от альтернативного описания, как и можно было ожидать исходя из замечания в предыдущем разделе. 


\section{5. ЗАКЛЮЧИТЕЛЬНЫЕ ЗАМЕЧАНИЯ}

Мы проанализировали, в какой степени альтернативные квантовые описания применимы к составным системам (состоящим из двух частей) на бесконечномерных комплексных гильбертовых пространствах. Мы показали, что только в случае, если альтернативная эрмитова структура связана с опорной посредством положительного оператора $F$ такого, что $F=F_{1} \otimes F_{2}$, проекцию на составляющие пространства можно выполнить вычислением частичных следов. Более того, мы показали, что энтропия фон Неймана сильно зависит от альтернативной эрмитовой структуры.

Мы надеемся, что настоящая работа может послужить основанием для дальнейших исследований положительных динамических отображений квантовых систем, состоящих из многих частей и наделенных альтернативными эрмитовыми структурами.

Благодарности. Автор благодарен профессору Дж. Мармо за плодотворные обсуждения.

\section{Список литературы}

[1] C. S. Gardner, J. M. Green, M. D. Kruskal, R. M. Miura, Phys. Rev. Lett., 19:19 (1967), 1095-1097; В. Е. Захаров, Л. Д. Фаддеев, Функи. анализ и его прил., 5:4 (1971), 18-27.

[2] F. Magri, J. Math. Phys., 19:5 (1978), 1156-1162; "A geometrical approach to the nonlinear solvable equations", Nonlinear Evolution Equations and Dynamical Systems, Proc. Meeting (Univ. Lecce, Lecce, 1979), Lecture Notes in Phys., 120, eds. M. Boiti et al., Springer, Berlin-New York, 1980, 233-263.

[3] S. De Filippo, G. Vilasi, G. Marmo, M. Salerno, Nuovo Cimento B, 83:2 (1984), 97-112.

[4] G. Marmo, E. J. Saletan, R. Schmid, A. Simoni, Nuovo Cimento B, 100:3 (1987), 297-317.

[5] B. A. Dubrovin, G. Marmo, A. Simoni, Modern Phys. Lett. A, 5:15 (1990), 1229-1234.

[6] J. F. Cariñena, J. Grabowski, G. Marmo, Internat. J. Modern Phys. A, 15:30 (2000), 4797-4810.

[7] A. Ibort, F. Magri, G. Marmo, J. Geom. Phys., 33:3-4 (2000), 210-228.

[8] G. Marmo, A. Simoni, F. Ventriglia, Rep. Math. Phys., 48:1-2 (2001), 149-157.

[9] F. Ventriglia, Modern Phys. Lett. A, 17:24 (2002), 1589-1599.

[10] G. Marmo, G. Morandi, A. Simoni, F. Ventriglia, J. Phys. A, 35:40 (2002), 8393-8406.

[11] G. Marmo, A. Simoni, F. Ventriglia, Rep. Math. Phys., 51:2-3 (2003), 275-285.

[12] G. Marmo, A. Simoni, F. Ventriglia, Internat. J. Modern Phys. A, 19:15 (2004), 2561-2578.

[13] G. Marmo, G. Scolarici, A. Simoni, F. Ventriglia, Note Mat., 23:2 (2004-2005), 173-181.

[14] Дж. Мармо, Дж. Сколаричи, А. Симони, Ф. Вентрилья, ТМФ, 144:2 (2005), 364-383.

[15] G. Marmo, G. Scolarici, A. Simoni, F. Ventriglia, Int. J. Geom. Methods Mod. Phys., 2:5 (2005), 919-937.

[16] G. Marmo, G. Scolarici, A. Simoni, F. Ventriglia, J. Phys. A, 38:17 (2005), 3813-3821.

[17] М. А. Наймарк, Нормированные кольца, Наука, М., 1968.

[18] V. I. Man'ko, G. Marmo, F. Zaccaria, E. C. G. Sudarshan, Rep. Math. Phys., 55:3 (2005), $405-422$.

[19] J. Grabowski, M. Kuś, G. Marmo, J. Phys. A, 38:47 (2005), 10217-10244.

[20] J. Grabowski, M. Kuś, G. Marmo, Open Syst. Inf. Dyn., 13:4 (2006), 343-362.

[21] F. G. Sholtz, H. B. Geyer, F. J. W. Hahne, Ann. Phys., 213:1 (1992), 74-101.

[22] C. A. Rodriguez, A. Shaji, E.C.G. Sudarshan, Dynamics of two qubits: decoherence and entanglement optimization protocol, arXiv: quant-ph/0504051. 